\title{
Correction to: Kinetic theory of acoustic-like modes in nonextensive pair plasmas
}

\author{
E. Saberian ${ }^{1,2}$ (1) $\cdot$ A. Esfandyari-Kalejahi ${ }^{3}$
}

Published online: 28 May 2019

(c) Springer Nature B.V. 2019

Correction to: Astrophys. Space Sci. (2014) 349: 799-811 https://doi.org/10.1007/s10509-013-1678-9

\begin{abstract}
Because of a misprint on numerical programming of one of the equations in the original publication, the existence of growing unstable acoustic-like modes in a $q$ nonextensive pair ion (or electron-positron) plasma had been predicted. It is confirmed that this picture is not appropriate and damping rate of acoustic-like modes in a $q$-nonextensive pair ion plasma is always negative and so resulting only in Landau damped modes. In this Erratum, the corrected and modified pattern of damping rate of acoustic-like modes is given in superextensive region with $q<1$ (the subsection involved inappropriate result) and the modified results are confirmed.
\end{abstract}

Keywords Electrostatic waves and oscillations - Plasma kinetic theory · Wave-particle interactions · Vlasov model

\section{Introduction}

In the original publication, in Sect. 4.2, the existence of growing unstable acoustic-like modes in a $q$-nonextensive

The online version of the original article can be found under https://doi.org/10.1007/s10509-013-1678-9.

$凶$ E. Saberian

e.saberian@neyshabur.ac.ir

1 Department of Physics, Faculty of Basic Sciences, University of Neyshabur, P.O. Box: 9319774446, Neyshabur, Iran

2 Research Department of Astronomy and Cosmology, University of Neyshabur, P.O. Box: 9319774446, Neyshabur, Iran

3 Research Institute for Applied Physics and Astronomy, University of Tabriz, Tabriz, Iran pair ion (or electron-positron) plasma had been predicted, where the spectral index $q$ takes the values in the region with $q<1$. As an Erratum, we confirm that this picture is not appropriate and damping rate of acoustic-like modes (Ion-acoustic waves) in a $q$-nonextensive pair ion plasma is always negative and so resulting only in Landau damped modes and no unstable modes. The origin of this misleading result was because of a misprint on numerical programming of one of the equations there, (Eq. (23) in the original publication), when two Figs. 4 and 6 had been plotted. Hereby, it is confirmed that all the analytical solutions in the original publication are true and the misleading result there had been arisen from a misprint in numerical programming of the mentioned equation. Furthermore, the other involved figures and conclusions are true and without mistake. In Sect. 2 of this Erratum, the corrected and modified pattern of Landau damping rate of acoustic-like modes is given in the superextensive region with $q<1$ (the subsection involved inappropriate result) and then the modified results and conclusions are confirmed in Sect. 3.

\section{The modified damping rate in region with $q<1$}

The fractional imaginary to real parts of acoustic-like modes in a $q$-nonextensive pair ion plasma for weakly damped modes was derived in Eq. (23) in the original publication as

$$
\begin{aligned}
\frac{\omega_{i}}{\omega_{r}}= & -\sqrt{\frac{\pi}{8}} L_{q}\left(\frac{1}{\left(k \lambda_{D}\right)^{2}\left(1+\frac{1}{\sigma}\right)+\left(\frac{1+q}{2}\right)}\right. \\
& \left.+3\left(\frac{2}{3 q-1}\right) \sigma\right)^{\frac{3}{2}}
\end{aligned}
$$




$$
\begin{aligned}
& \times\left\{\left[1-(q-1)\left(\frac{\frac{1}{2}}{\left(k \lambda_{D}\right)^{2}\left(1+\frac{1}{\sigma}\right)+\left(\frac{1+q}{2}\right)}\right.\right.\right. \\
& \left.\left.+\frac{3}{2}\left(\frac{2}{3 q-1}\right) \sigma\right)\right]^{\frac{2-q}{q-1}} \\
& +\frac{1}{\sigma^{\frac{3}{2}}}\left[1-(q-1)\left(\frac{\frac{1}{2 \sigma}}{\left(k \lambda_{D}\right)^{2}\left(1+\frac{1}{\sigma}\right)+\left(\frac{1+q}{2}\right)}\right.\right. \\
& \left.\left.\left.+\frac{3}{2}\left(\frac{2}{3 q-1}\right)\right)\right]^{\frac{2-q}{q-1}}\right\},
\end{aligned}
$$

where, $\sigma$ is the fractional temperature of positive to negative species, and the dimensionless $q$-dependent coefficient $L_{q}$ is given in two $q$-regions; superextensivity with $\frac{1}{3}<q<1$, and subextensivity with $q>1$ by the following expressions

$L_{q}=\frac{\Gamma\left(\frac{1}{1-q}\right)}{\Gamma\left(\frac{1}{1-q}-\frac{1}{2}\right)} \sqrt{1-q}, \quad$ for $\frac{1}{3}<q \leq 1$

$L_{q}=\left(\frac{1+q}{2}\right) \frac{\Gamma\left(\frac{1}{2}+\frac{1}{q-1}\right)}{\Gamma\left(\frac{1}{q-1}\right)} \sqrt{q-1} . \quad$ for $q \geq 1$

Unfortunately, in numerical analyzing of the mentioned equation, when we had numerically plotted this equation in Fig. 4 in the original publication for examining the damping rate of acoustic-like modes with respect to spectral index $q$ in region $\frac{1}{3}<q<1$, a mistyped factor $\Gamma\left(\frac{1}{q-1}-\frac{1}{2}\right)$ had been used in denominator of the expression of $L_{q}$ instead of the true factor $\Gamma\left(\frac{1}{1-q}-\frac{1}{2}\right)$. Note that, the expression given in Eq. (15a) in the original publication for $L_{q}$ in region $\frac{1}{3}<q<1$ is true (Eq. (2a) in this manuscript). The mentioned typo in programming codes made an incorrect pattern in Fig. 4 in the original publication and led to an incorrect conclusion, i.e., the existence of growing unstable acousticlike modes $\left(\frac{\omega_{i}}{\omega_{r}}>0\right)$ in the $q$-region $0.34 \lesssim q \lesssim 0.6$. The mentioned mistyped factor had been also used in plotting Fig. 6 in the original publication, where the pattern of Landau damping rate with respect to the wave number were discussed. So, two Figs. 4 and 6 in the original publication are not appropriate and have to be modified as follows.

The true numerical plotting of Eq. (1) with respect to spectral index $q$ in region $\frac{1}{3}<q<1$ leads to the pattern given in Fig. 1. Here, we have plotted the fractional imaginary to real parts of acoustic-like modes $\left(\frac{\omega_{i}}{\omega_{r}}\right)$ for typical values $k \lambda_{D}=0.1$ (long wavelength limit) and $\sigma=0.9$ (an insignificant temperature asymmetry). Figure 1 indicates that the damping rate of acoustic-like modes in a q-nonextensive pair plasma is always negative in region with $q<1$ and so resulting only in Landau damped modes and no unstable modes. Furthermore, it is found that the absolute value of $\frac{\omega_{i}}{\omega_{r}}$ increases with an increase in the spectral index $q$. This behavior also exist in region with $q>1$, as depicted in Fig. 5 of the original publication. Moreover, the fractional

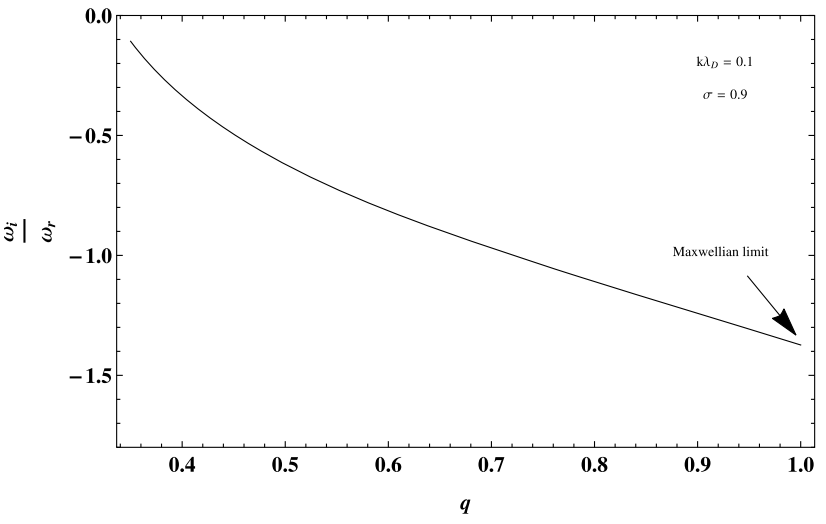

Fig. 1 The fractional imaginary to real parts of acoustic-like modes with respect to the spectral index $q$ in region with $q<1$ (superextensive region)

imaginary to real parts of acoustic-like modes $\left(\frac{\omega_{i}}{\omega_{r}}\right)$ in two regions with $q<1$ and $q>1$ tend smoothly to the same values in the limit $q \rightarrow 1$, i.e., at the Maxwellain limit (compare Fig. 1 here with Fig. 5 in the original publication at the limit $q \rightarrow 1$; for example they tend to the value -1.37 for $k \lambda_{D}=0.1$ and $\sigma=0.9$ ).

We emphasize that the occurrence of growing unstable electrostatic modes via a $q$-distribution is not possible. The reason is that the $q$-distribution is a decreasing function with velocity ( $q$-distribution is a single humped velocity distribution function). So, in the plasma modeled by a $q$-distribution there are on average more particles moving slightly slower than the wave than the particles moving slightly faster than the wave. In such a situation when the slower particles are accelerated by the wave, the energy of the wave is reduced and it leads to the Landau damping (Landau 1946). This picture shows that the $q$-distribution can't lead to the unstable modes. The interested reader may also refer to Saberian (2019), where the universality of stability with a $q$ distribution is discussed in detail as a general picture.

Furthermore, the revised and modified Landau damping rate with respect to the wave number has been depicted in Fig. 2 for some allowable values of the spectral index $q$. It is found that the damping rate is a sensitive function with respect to $q$ and it is always negative. Here, we have combined two cases of $q<1$ and $q>1$ for typical values $q=0.6,0.8,1,1.2,1.4$. The corresponding limit of $q \rightarrow 1$ indicates to a Maxwellian plasma. Moreover, Fig. 2 shows again that the absolute value of $\frac{\omega_{i}}{\omega_{r}}$ increases with $q$.

Here, we confirm that two Figs. 4 and 6 in the original publication have to be replaced by two Figs. 1 and 2 in this Erratum, and so the modified discussion and conclusions here have to be used for acoustic-like modes in a $q$-nonextensive pair ion plasma in region with $q<1$. We strongly confirm that all the analytical calculations in the original publication are true and the misleading results in plotting two Figs. 4 and 6 had been arisen from a mistype in 


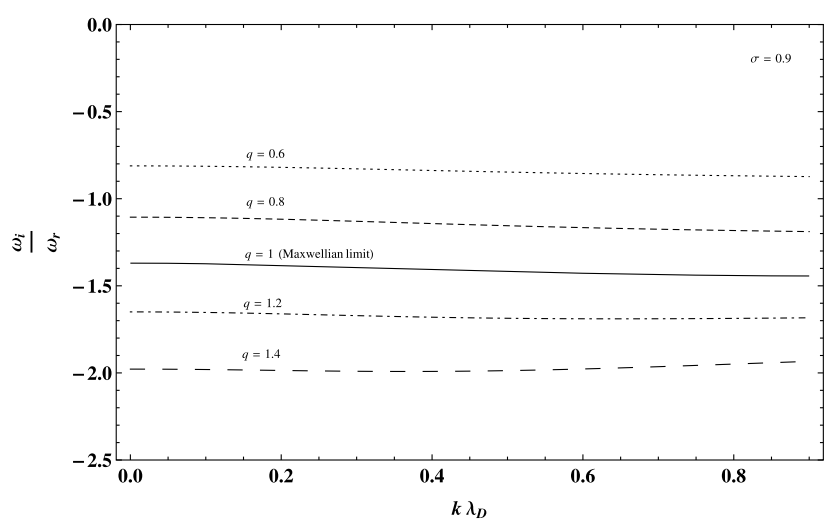

Fig. 2 The fractional imaginary to real parts of acoustic-like modes with respect to the wave number for some typical values of the spectral index $q$ in the both cases of $q<1$ and $q>1$

numerical programming of one of the equations there. The other sections, subsections and figures in the original publication are true. Summarizing this Erratum, the acoustic-like modes with a $q$-nonextensive distribution are only Landau damped and the growing unstable modes are not possible.

As a final and related point, it has been recently shown that the ion-acoustic waves (IAWs) in a pair-ion plasma are strongly damped (Saberian 2018). There, by applying the characteristic methods and solving the linearized VlasovPoisson equations, the full spectrum of plasma modes have been examined in a field-free pair plasma in the context of Tsallis statistics. There, it has been shown that the IAWs in a pair-ion plasma are strongly damped, and furthermore their damping time have been compared with the Langmuir-type modes. There it has been shown that Landau damping time for IAWs is negligible for most of wave lengths, and so these modes are heavily damped.

\section{Summary}

We resolved a misleading conclusion in the original publication, i.e., prediction of growing unstable acoustic-like modes in a $q$-nonextensive pair ion plasma, which had been arisen from a misprint in numerical programming of one of the equations there. True and modified pattern of the fractional imaginary to real parts of these modes $\left(\frac{\omega_{i}}{\omega_{r}}\right)$ was given in the region with $q<1$ and the modified results were confirmed.

It is found that $\frac{\omega_{i}}{\omega_{r}}$ in a $q$-nonextensive pair ion plasma is a sensitive function with respect to $q$, it is always negative and so resulting only in Landau damped modes and no unstable modes. Furthermore, it is found that the absolute value of $\frac{\omega_{i}}{\omega_{r}}$ increases with an increase in $q$.

Publisher's Note Springer Nature remains neutral with regard to jurisdictional claims in published maps and institutional affiliations.

\section{References}

Landau, L.D.: J. Phys. 10, 25 (1946)

Saberian, E.: Physica A 490, 289-299 (2018)

Saberian, E.: Phys. Rev. E 99, 017202 (2019) 